\title{
Neurological soft signs for discrimination between euthymic patients with bipolar I disorder and healthy controls
}

\author{
Eman Roshdy El Safy, Nagda Mohamed El Masry, Amany El Shabrawy and Ahmed Rafik Reda Abd Ellatif*
}

\begin{abstract}
Background: Neurological soft signs in remitted state of bipolar disorder may represent trait deficits and the aim of this study was to examine the extent of neurological soft signs in euthymic patients with bipolar I disorder as compared to healthy controls. We conducted this study in Zagazig University Hospital upon 60 subjects divided into two groups: euthymic patients with bipolar I disorder group (30 patients) and control group (30 healthy individuals). Assessment of neurological soft signs was performed through Neurological Evaluation Scale and the euthymic state was determined by Young Mania Rating Scale and Hamilton Depression Rating Scale.

Results: The euthymic patient group exhibited a significantly worse performance in the total Neurological Evaluation Scale and the whole four subtest scores than healthy control subjects. There was a statistically significant association between total neurological soft sign score and mood stabilizer therapy in the studied patients. The age of onset of the disorder was correlated to the total score of Neurological Evaluation Scale which is statistically significant. The best cutoff points of the total neurological soft signs score in the discrimination between the euthymic patient group and control group was 3.5 according to the receiver operating characteristic curve.
\end{abstract}

Conclusion: This study may emphasize the role of neurological soft signs as a sign of organic brain disorder; however, further studies may be able to extend our findings to explore the etiology and pathogenesis of bipolar I disorder.

Keywords: Neurological soft sign, Bipolar disorder, Euthymic patients

\section{Background}

Neurological soft signs (NSS) are described as sensory and motor performance non-localizing defects recognized through clinical examination. NSS involves observable sensory integration, motor coordination, and complicated motor sequencing defects that do not indicate a particular neurological disorder. Previous biological studies have demonstrated that these soft signs reflect functional disorders in the brain $[1,2]$.

Bipolar disorder (BD) is a significant cause of worldwide disability, an extremely common, often progressive disease with elevated treatment resistant, recurrence, and chronicity rates, and linked with elevated suicide and medical comorbidity rates of premature mortality $[3,4]$.

\footnotetext{
* Correspondence: ahmed.rafeek.10@gmail.com

Psychiatry Department, Faculty of Human Medicine, Zagazig University, Zagazig, Egypt
}

Despite various improvements in understanding bipolar disorder's etiology and underlying mechanisms, the results are still poor for many patients. As improvements can be produced with early management, appropriate early detection techniques such as biological peripheral biomarkers need to be developed $[5,6]$.

Neurological soft signs are suggested as a significant clinical tool for defining a subgroup of patients with neurodevelopmental predisposing factors that may have genetic and environmental roots [7]. Most extensive research has shown a greater incidence of neurological defects in schizophrenic patients; 92\% of schizophrenic patients, $52 \%$ of patients with affective disorder, and only $5 \%$ of control subjects have recorded neurological signs [8]. While NSS is the focus of a growing amount of schizophrenic research, comparatively few studies tackle the problem of NSS in bipolar I disorder [9]. 
It was suggested that NSS may be specifically associated with bipolar I disorder but not unipolar depression. The specificity of NSS expression reinforces the theory that NSS may play a potential role in early detection of the illness as a trait marker but not a state marker helping the discrimination of bipolar I disorder from disorders less likely to have a neurodevelopmental component [10].

Therefore, the aim of this study is to determine the scores of NSS in bipolar I disorder patients in remission state (euthymic patients) compared to healthy controls, in addition to explore correlation with some sociodemographic data and clinical characteristics of these euthymic patients.

\section{Methods}

A case-control study was conducted in Zagazig University hospitals at the psychiatry department. The study was conducted on 30 Egyptian subjects who were diagnosed to have bipolar I disorder in full remission (euthymic patients) (group A) which were selected by simple random sampling technique and on 30 apparently healthy persons as control group (group B). Recruitment was conducted between 1 October 2017 and 31 December 2018, and subjects who met the following eligibility criteria at enrollment were invited to participate: (1) both sexes were included: (2) age ranged from 20 to 50 years old: (3) patients fulfill the Diagnostic and Statistical Manual of Mental Disorders, fourth edition (DSM-IVTR) criteria for bipolar I disorder in full remission [11]: (4) all participants in both groups were matched for age, gender, and social classes. We excluded subjects with (1) age below 20 or above 50 years old, (2) patients having a history of medical, neurological, or other psychiatric disorders including substance use and alcohol, (3) patients presenting during the acute stage of the illness, and (4) refusal of participation. Ethical committee approval and written informed consent were obtained. All subjects were interviewed by a psychiatrist subjected to the following:

1. Collection of clinical and sociodemographic: All subjects were interviewed by a psychiatrist using semi-structured questionnaire specially developed for this study as designed to collect sociodemographic data as age, sex, marital status, education, occupation, age of onset of the disorder, duration of illness, number of admissions, and number of manic or depressive episodes.

2. Structured Clinical Interview for DSM-IV-TR Axis I Disorders (SCID-I) [12]: To exclude current psychiatric disorders of Axis I. It is a structured checklist interview that helps by its end to reach DSMIV-TR diagnosis as it contains a majority of psychiatric disorders with its subtypes. We used a clinical version for its simplicity and easy administration and because it does not differ on the basis of the type of subject being interviewed, so it can be used for the patients and healthy control subjects.

3. Young Mania Rating Scale (YMRS) [13]: To assess symptoms of mania and its severity. It is a highly reliable scale that consists of 11 items that screen all manic symptoms (some graded from zero to four and others from zero to eight). The rating depends mostly on the observation of the clinician and also on the words of the patients. This scale takes less than half an hour to end and to consider patient in euthymia, he should have a score lower than 12 .

4. Hamilton Depression Rating Scale (HDRS) [14]: To rate symptoms' severity of depression. It consists of 21 items. The scoring was based on the first 17 items that sum to give the final score while the remaining items are used to characterize manifestation of depression rather than the severity of it. This scale takes from 20 to $30 \mathrm{~min}$ to be completed and a patient is considered euthymic if he scored less than 12 .

5. The Neurological Evaluation Scale (NES) [15]: It is a structured instrument for the assessment of neurological soft signs. It includes representative items of sensory integration, motor coordination, sequencing of complete motor acts, and developmental reflexed. Each item is scored on a 3point scale: $0=$ no abnormality; $1=$ mild but definite impairment; and 2 = marked impairment except for the snout, suck, and glabellar reflexes which are scored either as a 0 or 2 . This scale was performed by a senior professor of psychiatry.

6. Fahmy and El-Sherbini's Social Classification Scale [16]: Participants were classified into social classes 1, 2, 3, and 4 according to an Egyptian classification developed by Fahmy and El-Sherbini. The classification is based on the following parameters: education of the father, education and work of the mother, income, crowding index, and sanitation.

Since the patients could not be asked to stop taking their medications-even temporarily-and these medications may affect some of the categories of NSS, we also tried to consider the analysis to assess any possible relationships between medication and NSS.

Therefore, we managed to categorize the type of medications into two main groups according to their pharmacological properties: (1) psychotropic drugs (antipsychotics and antidepressant) and (2) lithium and antiepileptic as mood-stabilizing agent.

The work has been carried out in accordance with the Code of Ethics of the World Medical Association (Declaration of Helsinki) for studies involving humans. 


\section{Statistical analysis}

All data were recorded and entered into a statistical package on a compatible computer and varied. The analysis was carried out using an SPSS, version 25 (SPSS Inc., Chicago, Illinois, USA). The results were tabulated, grouped, and statistically analyzed using the following tests: descriptive statistics (for quantitative data), as we used mean, median and range, and frequency with relative percentage (for qualitative data); the Student $t$ test, used to test for significance of an independent variable (to compare between two independent means); Pearson's chi-squares $\left(\chi^{2}\right)$ test was used to calculate difference between qualitative variables; Kruskal-Wallis (KW) test was used to calculate the difference between quantitative variables in more than two groups in not normally distributed data; Spearman's correlation coefficient $(r)$ was used to test correlation between NSS and continuous variables; the sensitivity, specificity, predictive values, accuracy, and likelihood ratios of NSS as an indicator of bipolar I disorder were calculated; and $P$ value used to indicate the level of significance was $P$ less than $0.05, P$ less than 0.01 for a highly significant difference, and $P$ less than 0.001 for a very highly significant difference.

\section{Results}

\section{Demographic data}

The demographic characteristics of the euthymic bipolar I patients and controls, as shown in Table 1, showed that there was no statistically significant difference between the studied groups in all items of sociodemographic characteristics.

\section{Clinical characteristics}

All patients were euthymic at the time of testing; their mean age of onset was 31.8 years old, with a mean duration of illness of 4.7 years, mean duration of longest admission of 4.7 weeks, and median number of previous episodes of 5 . Most of them, 93.3\%, received antipsychotics therapy and 90.0\% had lithium carbonate therapy (Table 2).

\section{Neurological soft signs (NSS) of the studied groups}

The total NSS score ranged from 3 to 12 in the patient group with a median of 6 , whereas it ranged from 0 to 3 in controls with a median score of 1 . The total NSS score was significantly higher in the patients compared to the controls $(P<0.001)$ which have been demonstrated in (Table 3 ) in detail. Increasing the educational

Table 1 Demographic characteristics of the studied groups

\begin{tabular}{|c|c|c|c|c|}
\hline Variables & Patient group $(n=30)$ & Control group $(n=30)$ & Test of sig. & $P$ \\
\hline \multicolumn{5}{|l|}{ Age (years) } \\
\hline$\pm \mathrm{SD}$ & $29.3 \pm 3.7$ & $29.9 \pm 3.5$ & $t, 0.6$ & 0.5 \\
\hline Range & $23.0-36.0$ & $21.0-37.0$ & & \\
\hline \multicolumn{5}{|l|}{ Sex } \\
\hline Males & $16(53.3 \%)$ & $15(50.0 \%)$ & $x^{2}, 0.6$ & 0.7 \\
\hline Females & $14(46.7 \%)$ & $15(50.0 \%)$ & & \\
\hline \multicolumn{5}{|l|}{ Marital status } \\
\hline Single & $14(46.7 \%)$ & $11(36.7 \%)$ & $x^{2}, 1.8$ & 0.9 \\
\hline Married & $9(30.0 \%)$ & $12(40.0 \%)$ & & \\
\hline Divorced & $6(20.0 \%)$ & $6(20.0 \%)$ & & \\
\hline Widow & $1(3.3 \%)$ & $1(3.3 \%)$ & & \\
\hline \multicolumn{5}{|l|}{ Education } \\
\hline Illiterate & $0(0.0 \%)$ & $0(0.0 \%)$ & $x^{2}, 8.7$ & 0.1 \\
\hline Elementary & $1(3.3 \%)$ & $3(10.0 \%)$ & & \\
\hline Preparatory & $5(16.7 \%)$ & $5(16.7 \%)$ & & \\
\hline Secondary & $22(73.3 \%)$ & $16(53.3 \%)$ & & \\
\hline High education & $2(6.7 \%)$ & $6(20.0 \%)$ & & \\
\hline \multicolumn{5}{|l|}{ Occupation } \\
\hline Working & $13(43.3 \%)$ & $21(70.0 \%)$ & $x^{2}, 4.4$ & 0.1 \\
\hline Not working & $17(56.7 \%)$ & $9(30.0 \%)$ & & \\
\hline \multicolumn{5}{|l|}{ Social class } \\
\hline Low & $13(43.3 \%)$ & $10(33.3 \%)$ & $x^{2}, 0.7$ & 0.7 \\
\hline Middle & $17(56.7 \%)$ & $20(66.7 \%)$ & & \\
\hline
\end{tabular}


Table 2 Clinical characteristics of the studied patients

\begin{tabular}{ll}
\hline Variables & Patients group $(n=30)$ \\
\hline Age of onset (years) & $31.8 \pm 4.9$ \\
Mean \pm SD & $22.0-42.0$ \\
Range & \\
Disease duration (years) & $4.7 \pm 1.3$ \\
Mean \pm SD & $3.0-7.0$ \\
Range & \\
Duration of longest admission (weeks) & $4.7 \pm 0.9$ \\
Mean \pm SD & $3.0-7.0$ \\
Range & \\
Number of previous episodes & 5 \\
Median & $3-7$ \\
Range & \\
Psychotropic therapy & \\
Antipsychotics & $28(93.3 \%)$ \\
Antidepressants & $2(6.7 \%)$ \\
Mood stabilizer drugs & \\
Lithium carbonate & $27(90.0 \%)$ \\
Sodium valproate & $3(10.0 \%)$ \\
\hline
\end{tabular}

${ }^{\mathrm{a}}$ Two patients had both antipsychotics and antidepressants therapies

level was correlated with a low occurrence of NSS which was shown in (Table 4).

NSS total score in discrimination between euthymic patients group and control group

The ROC curve analysis shows that NSS scores had a good differential efficacy for euthymic patients and healthy controls $(\mathrm{AUC}=0.99,(0.9-1.0))$. The optimal cutoff point for NSS scores was more than 3.5 (sensitivity $=96.7 \%$, specificity $=100 \%$ ) with positive predictive value $100 \%$ and accuracy $98.3 \%$ as shown in (Table 5) and (Fig. 1).

\section{Discussion}

While the sample of this research does not meet community sample requirements, its epidemiological and clinical features may make it cannot be a representative sample for bipolar disorder.

The slightly bigger proportion of males than females (53.3\% and $46.7 \%$, respectively) is very near to the usual frequency of mood disorders, as bipolar disorders are approximately equally common in males and females with a greater tendency towards hypomanic traits $[17,18]$.

Most patients were either divorced or single (66.7\%). Separate and divorced patients, either as a cause or as a consequence, have the largest rates of mood disorder. Presentation of patients in the unmarried single state can be explained by the early start of the disease which reduces their chance of being accepted for marriage due to the stigma of psychiatric disorders $[17,18]$.

The sample of this study showed a mean age at onset of the disorder of 31.8 years, which is consistent with other studies [19-21]. This, together with the mean duration of illness of 4.7 years and frequent hospitalization, gives an idea about the burden of this disease on the patients and their families especially their caregivers [22, 23].

\section{Neurological soft signs (NSS)}

Some researchers propose that NSS reflects a failure in the integration within or between sensory and motor systems [24], while others argue for deficits in neuronal

Table 3 Neurological soft signs (NSS) of the studied groups according to Neurological Evaluation Scale (NES)

\begin{tabular}{|c|c|c|c|c|}
\hline Neurological soft signs & Patients group $(n=30)$ & Control group $(n=30)$ & KW & $P$ \\
\hline \multicolumn{5}{|l|}{ Sensory integration } \\
\hline Median & 2.5 & 0 & 63.9 & $<0.001$ \\
\hline Range & $2-3$ & $0-2$ & & \\
\hline \multicolumn{5}{|l|}{ Motor coordination } \\
\hline Median & 2 & 0 & 41.3 & $<0.001$ \\
\hline Range & $0-3$ & $0-2$ & & \\
\hline \multicolumn{5}{|l|}{ Motor sequencing } \\
\hline Median & 1 & 0 & 23.1 & $<0.001$ \\
\hline Range & $0-3$ & $0-1$ & & \\
\hline \multicolumn{5}{|l|}{ Developmental reflexes } \\
\hline Median & 2 & 0 & 14.0 & $<0.001$ \\
\hline Range & $0-6$ & $0-2$ & & \\
\hline \multicolumn{5}{|l|}{ Total score } \\
\hline Median & 6 & 1 & 59.7 & $<0.001$ \\
\hline Range & $3-12$ & $0-3$ & & \\
\hline
\end{tabular}


Table 4 Correlation coefficients between total score of neurological soft signs and sociodemographic and clinical features of bipolar I patients

\begin{tabular}{ll}
\hline Sociodemographic and clinical features & Pearson coefficients \\
\hline Age & -0.14 \\
Educational level & -0.28 \\
Age at onset of BD & -0.02 \\
Duration of BD & -0.11 \\
Number of psychiatric hospitalizations & 0.04 \\
Number of mood episodes & 0.04 \\
Number of manic episodes & 0.01 \\
Number of depressive episodes & 0.07 \\
\hline
\end{tabular}

circuits involving subcortical structures (e.g., basal ganglia, brain stem, and limbic system) [25]. Some NSS has been suggested reflecting a more general "latent" neurological dysfunction; their research reinforces the concept of NSS as a marker of disordered neurodevelopment [26].

Although the presence of NSS has been widely documented in schizophrenia, in bipolar disorder, the same has not been achieved. In this research, bipolar patients showed a much worse performance on the NES than control subjects, thus strengthening the hypothesis that NSS can play a part in early identification of the disease, acting as trait markers [26-29].

From the above results, we can deduce that bipolar disorder, which can be regarded as one of the neurodevelopmental diseases, involves obvious neurological deficits in the neuronal circuits comprising the four categories of NSS, helping early detection of susceptible patients.

\section{Sociodemographic and clinical variables in relation to NSS}

In this study, there was no statistically significant gender correlation with NSS prevalence. This may be incompatible with comparable research on schizophrenia and the

Table 5 Diagnostic performance accuracy of total NSS score in discrimination between patients group and control group

\begin{tabular}{ll}
\hline Diagnostic performance & Total NSS score \\
\hline Cutoff point & More than 3.5 \\
Sensitivity & $96.7 \%$ \\
Specificity & $100 \%$ \\
Positive predictive value & $100 \%$ \\
Negative predictive value & $96.8 \%$ \\
Accuracy & $98.3 \%$ \\
Likelihood ratio positive & $\infty$ \\
Likelihood ratio negative & 0.00 \\
Area under the curve & $0.99(0.9-1.0)$ \\
$P$ & $<0.001(\mathrm{HS})$ \\
\hline
\end{tabular}

first psychotic episode, considering male gender as a predisposing factor for NSS development in schizophrenic patients [24, 30]. Also, there was no statistically significant relation between NSS and the age of patients, consistent with comparable NSS research in schizophrenia and psychosis [24, 30].

There was no correlation between the total NSS score and the age of the patients in this research and in accordance with other studies [31,32]. However, in neuroimaging research, an adverse correlation was discovered between BP age and gray matter volume [33, 34]. These modifications in histopathology could explain the earlier reported rise in NSS by age [35].

No significant correlation existed between total NSS score and gender. Similar findings were recorded [31, 31]. In female bipolar and schizophrenic patients, however, greater NSS scores were discovered [35]. Gender influence on NSS frequency in BP appears to be poorly studied and the findings are contradictory and inconsistent.

In this study, patients with low level of education had significantly higher scores of NSS which is in agreement with other studies $[33,21]$. This finding may give a clue to the NSS associating bipolar I disorder most probably as a trait marker [21].

However, Negash et al. have not found such a correlation [29]. Generally, educational failure in BP would be related to cognitive dysfunction rather than to premorbid intellectual deficit [29, 34].

No correlation was found between age at onset of BD and the score of NSS, which is consistent with the results of the only study that evaluated this association [35]. This describes the lack of correlation between the NSS and disease length. However, after 2 to 4 years of $\mathrm{BD}$ evolution, other trials revealed a reduction in NSS results [36, 37]. In essence, the improvement involved the sensory integration sub-score associated with improving mood symptoms [37].

No correlation existed between the NSS score and mood episode criteria. However, correlations between these state markers and trait markers such as the NSS are difficult to conclude. No correlation was discovered with either the number of episodes or their severity in line with this research [29].

NSS scores did not differ between patients whether treated by antipsychotics or not. This finding is in agreement with the results of other studies [28, 29]. Indeed, NSS are present in patients who have never been treated with antipsychotics and are not induced by antipsychotic medication [31, 38].

\section{Diagnostic performance accuracy of total NSS score}

In the assessment of the diagnostic performance accuracy of total NSS score in the current study according to the ROC curve, the total NSS score demonstrated a high 


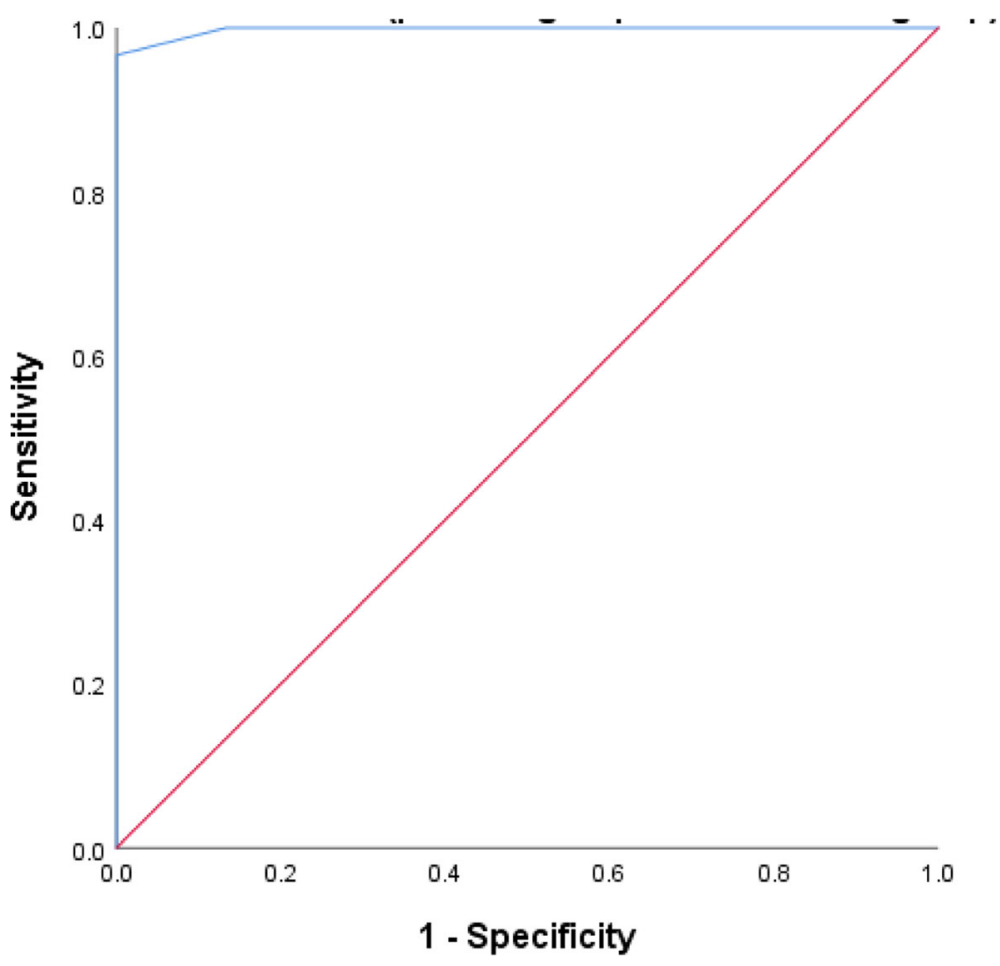

Fig. 1 ROC curve demonstrates total NSS score (patients group versus control group)

accuracy of being able to discriminate euthymic bipolar I disorder patients from healthy controls.

Results in (Table 5) revealed that the best cutoff point for the total NSS score to discriminate euthymic bipolar I disorder from control subjects was more than 3.5. This cutoff point provided sensitivity and specificity of $96.7 \%$ and $100 \%$, respectively, positive predictive value of $100 \%$, negative predictive value of $96.8 \%$, and diagnostic efficiency of $98.3 \%$ accurate in discriminating between euthymic bipolar I disorder patients from healthy controls indicating excellent diagnostic performance; also, area under the curve was 0.99 which was highly significant $(<0.001)$.

So, NSS as measured by NES can be considered as a further step in the diagnosis of bipolar I disorder and may be used as organic indicators in these patients which is supported by the study that was done by Noroozian et al. [35].

\section{Limitations}

There should be some limitations in our research. First, BP recruitment occurred solely in an outpatient psychiatric department and at the moment of incorporation they had to be euthymic. They may not generally represent the NSS frequency in BP. Furthermore, because there was a male predominance, our sample cannot be representative of $\mathrm{BD}$. This can be clarified by a selection bias because in our psychiatric department, male patients were more recruited. Second, using only hospital employees as healthy controls can result in a bias in selection. Also, because of the lack of patients on monotherapy, it was difficult to demonstrate the impact of each drug on the NSS individually. It must be acknowledged that examining drug-free patients is the most methodologically efficient method. However, it is highly uncommon to discover patients with proven bipolar disorder who are medication-free in clinical practice.

\section{Conclusions}

In conclusion, this study may emphasize the role of NSS as a sign of organic brain disorder that supports the hypothesis that bipolar disorder could be a neurodevelopmental disorder; however, further studies may be able to extend our findings to explore the etiology and pathogenesis of bipolar I disorder.

\section{Abbreviations \\ BP: Bipolar disorder; DSM-IV-TR: Diagnostic and Statistical Manual of Mental Disorders, fourth edition Text Revision; HDRS: Hamilton Depression Rating Scale; MoCA: Monterial Cognitive Assessment scale; NES: Neurological Evaluation Scale; NSS: Neurological soft signs; ROC curve: Receiver operating characteristic curve; YMRS: Young Mania Rating Scale}

\section{Acknowledgements \\ Not applicable}

Authors' contributions

ER and NM conceived and designed the study and analyzed the data. AE and AR wrote the first draft of the manuscript, interpreted the data, and 
wrote the final manuscript. All authors agreed with the results and conclusions of this article.

\section{Funding}

No funding sources.

\section{Availability of data and materials}

All data and materials are available.

\section{Ethics approval and consent to participate}

The study was approved by the Ethics Committee of the faculty of medicine, Zagazig University. All participants agreed to participate in the research, after receiving detailed information about the research. Written informed consent for participation in the study was obtained. The ethics reference number is not available.

\section{Consent for publication}

Not applicable.

\section{Competing interests}

The authors declare that they have no competing interests.

\section{Received: 29 October 2019 Accepted: 4 December 2019}

Published online: 19 February 2020

\section{References}

1. Zhao Q, Ma YT, Lui SS (2013) Neurological soft signs discriminate schizophrenia from major depression but not bipolar disorder. Prog NeuroPsychopharmacol Biol Psychiatry 43:72-78

2. Bombin I, Arango C, Buchanan RW (2005) Significance and meaning of neurological signs in schizophrenia: two decades later. Schizophr Bull 31(4): 962-977

3. Ghoryani M, Faridhosseini F, Talaei A (2019) Gene expression pattern of CCL2, CCL3, and CXCL8 in patients with bipolar disorder. J Res Med Sci 24(1):45-45

4. Plans L, Barrot C, Nieto E (2019) Association between completed suicide and bipolar disorder: a systematic review of the literature. J Affect Disord 242:111-122

5. Hosman C, Jane Llopis E, Saxena S (2004) Prevention of mental disorders: effective interventions and policy options: summary report

6. Gautam S, Jain A, Gautam M, Gautam A, Jagawat T (2019) Clinical practice guidelines for bipolar affective disorder (BPAD) in children and adolescents. Indian J Psychiatry 61(Suppl 2):294-305

7. Varambally S, Venkatasubramanian G, Gangadhar BN (2012) Neurological soft signs in schizophrenia - the past, the present and the future. Indian J Psychiatry 54(1):73-80

8. Romeo S, Chiandetti A, Siracusano A, Troisi A (2014) An exploratory study of the relationship between neurological soft signs and theory of mind deficits in schizophrenia. Psychiatry Res 218(1-2):7-11

9. Boks M, Liddle P, Burgerhof J, Knegtering R, van den Bosch RJ (2004) Neurological soft signs discriminating mood disorders from first episode schizophrenia, vol 110

10. Sagheer TA, Assaad S, Haddad G, Hachem D, Haddad C, Hallit S (2018) Neurological soft signs in bipolar and unipolar disorder: a case-control study. Psychiatry Res 261:253-258

11. Segal DL (2010) Diagnostic and Statistical Manual of Mental Disorders (DSMIV-TR), The Corsini Encyclopedia of Psychology, pp 1-3

12. First MB, Spitzer RL, Gibbon M, Williams JB. User's guide for the structured clinical interview for DSM-IV axis I disorders SCID-I: clinician version. American Psychiatric Pub; 1997

13. Young RC, Biggs JT, Ziegler VE (1978) A rating scale for mania: reliability, validity and sensitivity. Br J Psychiatry 133:429-435

14. Hamilton M (1960) A rating scale for depression. J Neurol Neurosurg Psychiatry 23:56-62

15. Buchanan RW, Heinrichs DW (1989) The Neurological Evaluation Scale (NES): a structured instrument for the assessment of neurological signs in schizophrenia. Psychiatry Res 27(3):335-350

16. Fahmy S, El-Sherbini AF (1988) Determining simple parameters for social classification for health research. Bull High Inst Pub Health 13:95-108
17. Weissman MM, Livingston B, Leaf PJ, Florio LP, Holzer C (1991) Affective disorders. In Psychiatric disorders in America: The Epidemiologic Catchment Area Study. In: Robins LN, Regier DA (eds) P53. Free Press, New York

18. Kessler RC, McGonagle KA, Zhao S (1994) Lifetime and 12-month prevalence of DSM-III-R psychiatric disorders in the United States. Results from the National Comorbidity Survey. Arch Gen Psychiatry 51(1):8-19

19. Ferrier IN, Mac MI, Young AH (2001) The search for the wandering thymostat: a review of some developments in bipolar disorder research. $\mathrm{Br}$ J Psychiatry 178(Suppl 41):S103-S106

20. Madsen AL, Vorstrup S, Rubin P (1999) Neurological abnormalities in schizophrenic patients: a prospective follow-up study 5 years after first admission. Acta Psychiatr Scand 100:119-125

21. Mrad A, Wassim Krir M, Ajmi I, Gaha L, Mechri A (2016) Neurological soft signs in euthymic bipolar I patients: a comparative study with healthy siblings and controls. Psychiatry Res 236:173-178

22. Heinrichs DW, Buchanan RW (1988) Significance and meaning of neurological signs in schizophrenia. Am J Psychiatr 145(1):11-18

23. Leask SJ, Done DJ, Crow TJ (2002) Adult psychosis, common childhood infections and neurological soft signs in a national birth cohort. $\mathrm{Br} J$ Psychiatry 181(5):387-392

24. El-Attar M, Okasha A, Sadek A, Beshry Z, Abdel-Ghany MO, El-Mahalawy N (1997) Schizophrenia as a neurodevelopmental disorder neurological soft signs and cognitive dysfunction. MD thesis. Ain Shams University, Egypt.

25. Dazzan P. Murray RM (2002) Neurological soft signs in first-episode psychosis: a systematic review. Br J Psychiatry Suppl 43:s50-s57

26. Manschreck T, Kopala L, Honer W (2003) Neurological comorbidity and features in schizophrenia. Medical IIIness and Schizophrenia: American Psychiatric Publishing, Inc., Arlington, pp 185-214

27. Madsen A, Vorstrup S, Rubin P, Larsen J, Hemmingsen R (1999) Neurological abnormalities in schizophrenic patients: a prospective follow-up study 5 years after first admission. Acta Psychiatr Scand 100(2):119-125

28. Boks MP, Liddle PF, Burgerhof JG, Knegtering R, Van den Bosch RJ (2004) Neurological soft signs discriminating mood disorders from first episode schizophrenia. Acta Psychiatr Scand 110(1):29-35

29. Negash A, Kebede D, Alem A (2004) Neurological soft signs in bipolar I disorder patients. J Affect Disord 80(2-3):221-230

30. Brambilla P, Harenski K, Nicoletti M (2001) Differential effects of age on brain gray matter in bipolar patients and healthy individuals. Neuropsychobiology. 43(4):242-247

31. Chen EY-H, Hui CL-M, Dunn EL-W (2005) A prospective 3-year longitudinal study of cognitive predictors of relapse in first-episode schizophrenic patients. Schizophr Res 77(1):99-104

32. Goswami U, Sharma A, Khastigir U (2006) Neuropsychological dysfunction, soft neurological signs and social disability in euthymic patients with bipolar disorder. Br J Psychiatry 188(4):366-373

33. Compton MT, Bollini AM, Mack LM (2007) Neurological soft signs and minor physical anomalies in patients with schizophrenia and related disorders, their first-degree biological relatives, and non-psychiatric controls. Schizophr Res 94(1-3):64-73

34. Kapczinski F, Vieta E, Andreazza AC (2008) Allostatic load in bipolar disorder: implications for pathophysiology and treatment. Neurosci Biobehav Rev 32(4):675-692

35. Noroozian M, Amini H, Faridhosseini Md F, Irandoost P, Saghaie T (2009) Neurological soft signs: a further step in the diagnosis of bipolar-I disorder? vol 4

36. Mayoral M, Bombín I, Castro-Fornieles J (2012) Longitudinal study of neurological soft signs in first-episode early-onset psychosis. J Child Psychol Psychiatry 53(3):323-331

37. Whitty P, Clarke M, McTigue O (2006) Diagnostic specificity and predictors of neurological soft signs in schizophrenia, bipolar disorder and other psychoses over the first 4 years of illness. Schizophr Res 86(1-3):110-117

38. Scheffer RE (2004) Abnormal neurological signs at the onset of psychosis. Schizophr Res 70(1):19-26

\section{Publisher's Note}

Springer Nature remains neutral with regard to jurisdictional claims in published maps and institutional affiliations. 